\title{
WORST SHAPES OF IMPERFECTIONS FOR SPACE TRUSSES WITH MULTIPLE GLOBAL AND LOCAL BUCKLING MODES
}

\author{
Ralf Peek \\ Department of Civil Engineering, 2340 G. G. Brown Laboratory, The University of Michigan, \\ Ann Arbor, MI 48109, U.S.A.
}

(Received 4 August 1992; in revised form 16 January 1993)

\begin{abstract}
The initial postbuckling behavior and imperfection sensitivity of truss-type structures in which the joints do not transmit moments is determined by means of the Lyapunov-SchmidtKoiter approach. Such structures possess local buckling modes involving the buckling of individual members, and global modes involving axial deformations of all members but no member buckling. Of particular interest is the case in which through optimization (or otherwise) a number of local and global modes are coincident. The worst shape of imperfection for this case is determined from the bifurcated equilibrium branch on which the load drops most rapidly. It is shown that this critical bifurcated branch initially involves the buckling of one member only. The general theory is illustrated by a number of examples involving two- and three-dimensional lattice columns. The leading order asymptotic results for these examples are compared to exact results obtained by tracking the appropriate equilibrium branch numerically.
\end{abstract}

\section{NOMENCLATURE}

$b_{(m)}=x_{(m)} / l$

$e_{(m)}=l-L=$ member elongation

$e_{\mathrm{c}(m)}$

$\bar{e}_{\mathrm{e}(m)}=\left(\ldots, \bar{e}_{(m)}, \ldots\right)$
$\bar{e}_{(m)}=L-L$

$E A$

$E I$

$G$

$L=L_{(m)}=L+\bar{e}_{(m)}$

$L=L_{(m)}=\left\|\bar{X}_{(m)}\right\|$

$l=l_{(m)}=\left\|x_{(m)}\right\|$

$M$

$M_{0}$

$M_{1}$

$n(\cdot)$

$N_{\mathrm{c}(m)} \equiv-\phi_{(m), \mathrm{c}}^{\mathrm{c}}$

$P(\lambda)$

$s=\sqrt{E I /\left(l^{2} E A\right)}$

$u=(v, w)$

$\bar{u}=(\bar{v}, \bar{e}, \bar{W})$

$\bar{u}_{(m)}=\left(\bar{v}_{(m)}, \bar{e}_{(m)}, W_{(m)}\right)$

$\bar{v}$

$v_{(m)}$

$\bar{v}_{(m)}$

$w=\left(\ldots, w_{(m)}, \ldots\right)$

$w_{(m)}$

$W_{(m)}$

$X$

$X=X+v$ unit vector along member direction in the deformed configuration

change in member length from stress-free condition to deformed configuration member shortening at criticality

imperfections in lengths of members

imperfection in member length for member $m$ (zero if structure is stress-free in reference configuration for imperfect structure)

axial rigidity of member

flexural rigidity of member

set of all global mode identifiers

length of member $m$ in stress-free condition

length of member $m$ in reference configuration of imperfect structure

length of member $m$ in deformed configuration (straight distance from joint to joint)

set of all local mode identifiers

set of local mode identifiers corresponding to inactive local modes (members that do not buckle)

set of local mode identifiers corresponding to active local modes (members that buckle)

number of elements in a set which appears in parentheses as an argument critical axial load for member $\boldsymbol{m}$

linear operator representing the work done by loads applied at the joints member slenderness ratio

vector describing the configuration of the structure at any time

vector describing the imperfections of the structure

vector describing the imperfections affecting an individual member

collection of all joint displacement vectors

vector of imperfections in joint coordinates

difference $\dagger$ in the joint displacement vectors at each end of member $m$

difference in imperfections in joint coordinates at each end of member $\boldsymbol{m}$

collection of all member buckling displacements

member buckling displacement for member $m$

vector describing imperfections involving out-of-straightness of members, and end eccentricities

vector describing out-of-straightness and end eccentricities for member $m$ vector containing all joint coordinates for the perfect structure in the undeformed configuration

vector containing all joint coordinates for the imperfect structure in its reference configuration

† Such differences in displacements at each end of the member should be taken by defining a beginning and end for each member, and always subtracting the displacement at the beginning from that at the end. 
$x=\bar{X}+v=X+\bar{v}+v$

$X_{(m)}$

$\bar{X}_{(m)}^{(m)}=X_{(m)}+v_{(m)}$

$x_{(m)}=\bar{X}_{(m)}+v_{(m)}=X_{(m)}+\bar{v}_{(m)}+v_{(m)}$ $\varepsilon_{\mathrm{c}}$

$\delta(\cdot), \delta_{1}(\cdot), \delta_{,}(\cdot)$, etc

$\delta_{I J}$

$\phi_{(m)}=\phi_{(m)}\left(e_{(m)}, w_{(m)}, \tilde{u}_{(m)}\right)$ vector containing all joint coordinates for the structure in its deformed configuration

difference in joint coordinates $X$ at each end of member $m$

difference in joint coordinates $\bar{X}$ at each end of member $m$

difference in joint coordinates $x$ at each end of member $m$

strain in member at bifurcation point

variations of $(\cdot)$

Kronecker delta

strain energy function for member $m$.

\section{INTRODUCTION}

Stability considerations are often important in the design of truss-type structures in which the moment transfer at the joints can be neglected. When member buckling controls the design of certain members, cost or weight minimization usually leads to a design in which none of these members has excess capacity. As a result, several members reach their buckling load at the same time. Thus the system as a whole has a number of coincident local buckling modes. This multiple bifurcation problem involving local-local mode interaction was considered in an earlier companion paper (Peek and Triantafyllidis, 1992).

For a variety of slender or thin structures, global buckling modes can also develop. These involve axial deformation of all members but no member buckling. For example, lattice columns can exhibit an overall Euler-type buckling mode. Also, lattice domes behave much like thin shells (Forman and Hutchinson, 1970). Thus their global buckling modes are similar to those of thin shells. This can lead to a number of coincident or closely spaced global modes. Optimization tends to produce a condition in which local and global modes are coincident. Such structures experience symmetry-breaking mode interactions, and, as a result, are very imperfection sensitive. Although existing methods allow the imperfect structure to be analysed, the results are strongly dependent on the shape of the imperfection. It is therefore important to determine the worst shape of imperfection.

Here general results (Koiter, 1976; Triantafyllidis and Peek, 1992) are applied to determine the worst imperfection shape from the bifurcated equilibrium branch for the perfect structure on which the load drops most rapidly. The multiple bifurcation problem involving both local and global modes is approached from the point of view of the Lyapunov-Schmidt-Koiter initial postbuckling analysis. The method allows the worst shape for a small imperfection to be determined for any two- or three-dimensional truss-type structure with coincident local and global modes. It also provides a simple relationship between the magnitude of the imperfection and the corresponding load drop (defined as the difference between the bifurcation load for the perfect structure and the load at the first limit point for the imperfect structure). In addition, two interesting qualitative results are obtained: (1) that all bifurcated equilibrium branches are unstable in the vicinity of the bifurcation point, and (2) that the bifurcated equilibrium branch on which the load drops most rapidly involves buckling of one member only.

The second of these qualitative results has important consequences: it implies that the worst imperfection shape [in the sense of Koiter (1976), or Triantafyllidis and Peek (1992)] involves only one imperfect member. It also simplifies the problem of finding the critical bifurcated branch (and worst imperfection shape), since only bifurcated branches involving buckling of one member need to be considered. As a result the computational effort is only linearly increasing in the number of local modes, whereas it is exponentially increasing in the number of coincident global modes.

Much of the notation in this paper parallels that in the companion paper (Peek and Triantafyllidis, 1992). Nevertheless, to make this paper self-contained, all symbols are defined in the Nomenclature section. In addition, any new notation (not in the companion paper) is defined in the text as it first appears.

\section{FORMULATION AND EQUILIBRIUM BRANCHES FOR PERFECT STRUCTURE}

It is assumed that :

(i) The structure remains elastic, with member strain energy given by 


$$
\phi_{(m)}=\int_{0}^{L} \frac{1}{2}\left(E A \varepsilon^{2}+E I \kappa^{2}\right) \mathrm{d} S,
$$

where $\varepsilon=(\mathrm{d} s-\mathrm{d} S) / \mathrm{d} S$ is the engineering axial strain in the member, $\mathrm{d} s(\mathrm{~d} S)$ is length increment along the centroidal axis of the member in the deformed (undeformed) configuration, $\kappa=(\mathrm{d} \theta-\mathrm{d} \bar{\theta}) / \mathrm{d} S$ is the bending deformation, and $\theta(\bar{\theta})$ represents the angle in radians between the axis of the member and a line connecting the member end points in the deformed (undeformed) configuration. The undeformed configuration is assumed to be stress free, but does incorporate geometric imperfections. Thus $\theta$ includes the rotation associated with the imperfection as well as that duc to deformation of the structurc, whercas $\bar{\theta}$ includes only rotations associated with the imperfection.

(ii) External loads are applied at the joints only, and their magnitude and direction depends on the load parameter $\lambda$ only, and not on the deformations of the structure.

(iii) All joints are pinned with zero moment transfer across the pins. However members may enter the joint cccentrically. Such eccentricity results in an end moment on the eccentric member only, and does not affect other members. Throughout this paper the terms "joints" or "end points of a member" refer to the location of the pins.

With these assumptions, the total potential energy of the structure can be written as

$$
\phi(u, \lambda, \bar{u})=\phi(v, w, \lambda, \bar{u})=\sum_{m} \phi_{(m)}\left(e_{(m)}, w_{(m)}, \bar{u}_{(m)}\right)-P(\lambda) v
$$

where $\phi_{(m)}$ is the strain energy in member $m, u=(v, w)$ is a vector describing the configuration of the structure at any time, with $v$ denoting the joint displacements, and $w=\left(\ldots, w_{(m)}, \ldots\right)$ denoting the member buckling displacements.

Specifically, $w_{(m)}$ is a displacement parameter that arises in the postbuckling analysis of member $m$ by the Lyapunov-Schmidt-Koiter approach as described in Appendix B of the companion paper (Peek and Triantafyllidis, 1992). Briefly, the transverse displacement (as measured from a line connecting the end points of the member) is decomposed into two parts: One which is proportional to the (sinusoidal) member buckling mode, and a higher order part that is orthogonal to the member buckling mode. The displacement parameter $w_{(m)}$ is the maximum transverse displacement associated with the first part. It is also an approximation to the total maximum transverse displacement.

The imperfections are described by a vector $\bar{u}=\left(\ldots, \bar{u}_{(m)}, \ldots\right)$, where $\bar{u}_{(m)}$ describes the imperfections affecting member $m$. For the moment the perfect structure is considered so that $\bar{u}=0$.

Evaluating the member strain energy and its derivatives with respect to $e_{(m)}$ and $w_{(m)}$ on the principal equilibrium branch for the perfect member gives

$$
\begin{gathered}
\phi_{(m)}(e, 0,0)=\frac{1}{2} \frac{E A}{L} e^{2}, \quad \phi_{(m), e}(e, 0,0)=\frac{E A}{L} e, \\
\phi_{(m), e e}(e, 0,0)=\frac{E A}{L}, \quad \phi_{(m), e e e}(e, 0,0)=0, \\
\phi_{(m), w}(e, 0,0)=\phi_{(m), e w}(e, 0,0)=\phi_{(m), e e w}(e, 0,0)=\phi_{(m), w w w}(e, 0,0)=0 .
\end{gathered}
$$

The bifurcation point for the member occurs at $\left(e_{(m)}, w_{(m)}, \bar{u}_{(m)}\right)=\left(-e_{\mathrm{c}(m)}, 0,0\right)$, with $e_{\mathrm{c}(m)}=\varepsilon_{\mathrm{c}} L, \varepsilon_{\mathrm{c}}=(\pi s)^{2}$, and $s=\sqrt{E I /\left(l^{2} E A\right)}$ is the slenderness ratio of the member. Additional derivatives of the member strain energy evaluated at criticality are given by

$$
\phi_{(m), w w}^{\mathrm{c}}=0, \quad \phi_{(m), e w w}^{\mathrm{c}}=\frac{\pi^{2}}{2} \frac{E A}{L^{2}}
$$

where the superscript $\mathrm{c}$ indicates that the quantity is evaluated at the bifurcation or critical 
point. These results for a single member are based on large displacement theory, with terms of the order $\varepsilon_{\mathrm{c}}^{2}$ in the final results being neglected when compared to unity (Peek and Triantafyllidis, 1992, Appendix B). However, it is only in the fourth and higher energy derivatives that significant differences between the large and moderate deflection theory appear.

The member elongation $e_{(m)}$ and its Frechet derivatives with respect to joint displacements $v$ are given by

$$
\begin{gathered}
e_{(m)}=l-L=\left\|x_{(m)}\right\|-\left\|X_{(m)}\right\|=\left\|X_{(m)}+v_{(m)}\right\|-\left\|X_{(m)}\right\|, \\
e_{(m), v} \delta v=b_{(m)} \cdot \delta v_{(m)}, \quad b_{(m)}=\frac{1}{l} x_{(m)}, \\
e_{(m), v v} \delta_{2} v \delta_{1} v=\frac{1}{l}\left\{\delta_{2} v_{(m)} \cdot \delta_{1} v_{(m)}-\left(b_{(m)} \cdot \delta_{2} v_{(m)}\right)\left(b_{(m)} \cdot \delta_{1} v_{(m)}\right)\right\} \\
e_{(m), v v v} \delta_{3} v \delta_{2} v \delta_{1} v=\frac{1}{l^{2}}\left\{3\left(b_{(m)} \cdot \delta_{3} v_{(m)}\right)\left(b_{(m)} \cdot \delta_{2} v_{(m)}\right)\left(b_{(m)} \cdot \delta_{1} v_{(m)}\right)\right. \\
-\left(b_{(m)} \cdot \delta_{3} v_{(m)}\right)\left(\delta_{1} v_{(m)} \cdot \delta_{2} v_{(m)}\right)-\left(b_{(m)} \cdot \delta_{2} v_{(m)}\right)\left(\delta_{3} v_{(m)} \cdot \delta_{1} v_{(m)}\right) \\
\left.-\left(b_{(m)} \cdot \delta_{1} v_{(m)}\right)\left(\delta_{2} v_{(m)} \cdot \delta_{3} v_{(m)}\right)\right\}
\end{gathered}
$$

where $\delta v, \delta_{1} v$, etc. and $\delta w, \delta_{1} w$, etc. denote variations in the displacements $v$ and $w$, respectively; and $\delta v_{(m)}, \delta_{1} v_{(m)}$, etc. are the difference in the variations in joint displacement at each end of member $m$.

The equilibrium conditions (stationarity of the potential energy) are

$$
\begin{aligned}
\phi_{. v} \delta v & =\sum_{m} N_{(m)} e_{(m), v} \delta v-P(\lambda) \delta v=0, \\
\phi_{, w} \delta w & =\sum_{m} \phi_{(m), w} \delta w_{(m)}=0,
\end{aligned}
$$

where $N_{(m)} \equiv \phi_{(m) . e}$ is the axial force in member $m$. From eqn (5) it is seen that the equilibrium condition [eqn (12)] is satisfied when all member buckling displacements are zero (i.e. when $w=0$ ). As a result, the principal equilibrium branch for the perfect structure can be represented as

$$
\stackrel{\circ}{u}(\lambda)=(\stackrel{o}{v}(\lambda), 0)
$$

where "o" placed above any symbol denotes evaluation on the principal branch.

The higher derivatives of the total potential energy with respect to the displacements $u=(v, w)$ evaluated on the principal equilibrium branch for the perfect structure are

$$
\begin{aligned}
& \stackrel{\circ}{\phi}_{, v} \delta_{2} v \delta_{1} v=\sum_{m}\left\{\left(\frac{E A}{L}-\frac{N}{l}\right)_{(m)}\left(b_{(m)} \cdot \delta_{1} v_{(m)}\right)\left(b_{(m)} \cdot \delta_{2} v_{(m)}\right)+\left(\frac{N}{l}\right)_{(m)} \delta_{1} v_{(m)} \cdot \delta_{2} v_{(m)}\right\} \\
& \stackrel{\circ}{\phi}, v w_{, w} \delta v=0, \\
& \stackrel{\circ}{\phi}_{, w w} \delta_{2} w \delta_{1} w=\sum_{m} \phi_{(m), w w} \delta_{2} w_{(m)} \delta_{1} w_{(m)}, \\
& \stackrel{\circ}{\phi}_{, v v v} \delta_{3} v \delta_{2} v \delta_{1} v=\sum_{m} \frac{1}{l}\left(\frac{E A}{L}-\frac{N}{l}\right)_{(m)}\left\{\left(b_{(m)} \cdot \delta_{1} v_{(m)}\right)\left(\delta_{2} v_{(m)} \cdot \delta_{3} v_{(m)}\right)\right. \\
& +\left(b_{(m)} \cdot \delta_{2} v_{(m)}\right)\left(\delta_{3} v_{(m)} \cdot \delta_{1} v_{(m)}\right)+\left(b_{(m)} \cdot \delta_{3} v_{(m)}\right)\left(\delta_{1} v_{(m)} \cdot \delta_{2} v_{(m)}\right) \\
& \left.-3\left(b_{(m)} \cdot \delta_{1} v_{(m)}\right)\left(b_{(m)} \cdot \delta_{2} v_{(m)}\right)\left(b_{(m)} \cdot \delta_{3} v_{(m)}\right)\right\}
\end{aligned}
$$




$$
\begin{aligned}
& \stackrel{0}{\phi}, v v w \delta w \delta_{2} v \delta_{1} v=0, \\
& \phi_{, v w w}^{\mathrm{c}} \delta_{2} w \delta_{1} w \delta v=\sum_{m} \frac{\pi^{2}}{2}\left(\frac{E A}{L^{2}}\right)_{(m)} \delta_{1} w_{(m)} \delta_{2} w_{(m)}\left(b_{(m)} \cdot \delta v_{(m)}\right), \\
& \stackrel{\circ}{\phi}, w w w_{3} \delta_{3} \delta_{2} w \delta_{1} w=0 \text {. }
\end{aligned}
$$

In eqns (14)-(20), the "o" placed above $\phi$ indicates that the energy derivatives are evaluated on the principal equilibrium branch for the perfect structure, whereas the superscript "c" [as in eqn (19)] denotes evaluation at the bifurcation point.

In view of eqn (15), it is seen that the buckling modes $\stackrel{(i)}{u} \equiv(\stackrel{i}{v}, \stackrel{(i)}{w})$ are of two types: Global modes of the form $\stackrel{(\alpha)}{u}=(\stackrel{(\alpha)}{v}, 0)$, involving axial deformations of all members, but no member buckling, for which

$$
\phi_{, v v}^{\mathrm{c}} \stackrel{(\alpha)}{v} \delta v=0 \quad \forall \delta v, \quad \forall \alpha \in G
$$

and local modes of the form $\stackrel{(i)}{u}=(0, \stackrel{(i)}{w})$, involving buckling of an individual member, but no joint displacements, for which

$$
\phi_{, w w}^{\mathrm{c}} \stackrel{(i)}{w} \delta w=0 \quad \forall \delta w, \quad \forall i \in M
$$

It is assumed that a number of global buckling modes, $\alpha \in G$, and local buckling modes, $i \in M$, occur at the same value of the load parameter, $\lambda=\lambda_{\text {c }}$. For convenience the local mode identifiers, $i \in M$, are taken to coincide with the member numbers. Thus buckling mode $i \in M$ involves buckling of member $i$ only, and can be represented as

$$
\stackrel{(i)}{w}=\left(\ldots, \stackrel{(i)}{w}_{(m)}, \ldots\right), \quad \stackrel{(i)}{w}_{(m)}=0 \quad \forall m \neq i
$$

Greek letters as mode identifiers always refer to global modes, lower case roman letters refer to local modes, and upper case roman letters refer to either local or global modes, i.e.

$$
\alpha, \beta, \gamma, \ldots \in G, \quad i, j, k, \ldots \in M, \quad I, J, K, \ldots \in M \cup G
$$

The modes are orthonormalized so that

$$
\left[\frac{\mathrm{d}}{\mathrm{d} \lambda} \phi_{, u u}(\stackrel{0}{u}(\lambda), \lambda, 0) \stackrel{(J)}{u} \stackrel{(J)}{u}\right]_{\lambda=\lambda_{\mathrm{c}}}=-\phi_{0} \delta_{I J} \quad \forall I, J \in M \cup G
$$

where the normalization constant $\phi_{0}$ is taken to be

$$
\phi_{0}=\frac{\pi^{2} N_{\mathrm{c}(0)} L_{(0)}}{2 \lambda_{\mathrm{c}}}
$$

and $N_{\mathrm{c}(0)}$ and $L_{(0)}$ represent the critical axial load and length of some reference member. For the local modes, this normalization condition is satisfied if

$$
\stackrel{i}{w}_{(i)}=\sqrt{L_{(i)} L_{(0)} N_{\mathrm{c}(0)} /\left(-\lambda_{\mathrm{c}} \dot{N}_{(i)}\right)}
$$

where a dot placed above any quantity indicates that this quantity should be evaluated on the principal branch (as a function of $\lambda$ only), then differentiated with respect to $\lambda$ and evaluated at $\lambda=\lambda_{\mathrm{c}}$.

The normalization described by eqn (26) ensures that for linear prebuckling behavior (i.e. when $\lambda_{\mathrm{c}} \dot{N}_{(i)}=-N_{\mathrm{c}(i)}$ ), the unit member buckling displacement coincides with the length 
of the member (i.e. $\stackrel{(i)}{w}_{(i)}=L_{(i)}$ ) for any member whose properties $N_{\mathrm{c}(i)}$ and $L_{(i)}$ coincide with those of the reference member.

For the global modes, the orthonormalization condition [eqn (25)] reduces to

$$
\phi_{, v v v}^{\mathrm{c}} \stackrel{(\alpha)(\beta)}{v} v \dot{v}=-\phi_{0} \delta_{\alpha \beta}
$$

According to the general theory [as described for instance in Triantafyllidis and Peek (1992)], the bifurcated equilibrium branches for the perfect structure can be described parametrically by the following expressions for the load parameter $\lambda$ and the displacements as a function of a path parameter, $\xi$ :

$$
\lambda=\lambda_{\mathrm{c}}+\lambda_{1} \xi+0\left(\xi^{2}\right), \quad u=\stackrel{0}{u}(\lambda)+\xi \sum_{I \in M \cup G} \alpha_{I} \stackrel{(I)}{u}+0\left(\xi^{2}\right),
$$

where $\lambda_{1}$ and $\alpha_{I}$ are determined from the conditions

$$
\begin{gathered}
-\lambda_{1} \alpha_{I}+\frac{1}{2} \sum_{J . K \in G \cup M} \phi_{I J K} \alpha_{J} \alpha_{K}=0 \quad \forall I \in G \cup M, \\
\sum_{I \in G \cup M}\left(\alpha_{l}\right)^{2}=1
\end{gathered}
$$

and $\phi_{I J K}$ are third order postbuckling coefficients defined as

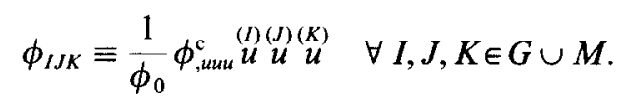

Evaluating these coefficients for the various combinations of local and global modes gives

$$
\begin{aligned}
& \phi_{\alpha \beta \gamma}=\frac{1}{\phi_{0}} \phi_{. v v v}^{\mathrm{c}} \stackrel{(\alpha)(\beta)(\gamma)}{v} v \quad \forall \alpha, \beta, \gamma \in G \\
& =\sum_{m} \frac{1}{\phi_{0} l}\left(\frac{E A}{L}-\frac{N}{l}\right)_{(m)}\left\{\stackrel{(\alpha)}{e}_{(m)} \stackrel{(\beta)}{v}_{(m)} \cdot \stackrel{(\gamma)}{v}_{(m)}+\stackrel{(\beta)}{e}_{(m)} \stackrel{(p)}{v}_{(m)} \cdot \stackrel{(\alpha)}{v}_{(m)}+\stackrel{(\vartheta)}{e}_{(m)} \stackrel{(\alpha)}{v}_{(m)} \cdot \stackrel{(\beta)}{v}_{(m)}\right. \\
& \left.-3 \stackrel{(\alpha)}{e}_{(m)}{ }_{(\beta)}^{(\beta)}{ }_{(m)} \stackrel{(\gamma)}{e}{ }_{(m)}\right\}, \\
& \phi_{\alpha \beta i}=\phi_{\alpha i \beta}=\phi_{i \alpha \beta}=\frac{1}{\phi_{0}} \phi_{. v v w}^{\mathrm{c}} \stackrel{(\alpha)(\beta)(i)}{v} \underset{w}{w}=0 \quad \forall(\alpha, \beta, i) \in G \times G \times M, \\
& \phi_{\alpha i j}=\phi_{i \alpha j}=\phi_{i j \alpha}=\frac{1}{\phi_{0}} \phi_{(i), e w w}\left(\stackrel{(i)}{w_{(i)}}\right)^{2}\left(e_{(i), v}{ }^{(\alpha)}\right) \delta_{i j} \quad \forall(\alpha, i, j) \in G \times M \times M \\
& =-\left(\stackrel{(\alpha)}{N}_{(i)} / \dot{N}_{(i)}\right) \delta_{i j} \\
& \phi_{i j k}=0 \quad \forall i, j, k \in M \text {, }
\end{aligned}
$$

where a global mode identifier placed in parentheses over any entity denotes the Frechet Derivative of this entity with respect to joint displacements $v$ operating on the corresponding modeshape. Thus,

$$
\stackrel{(\alpha)}{e}_{(m)}=e_{(m), v} \stackrel{(\alpha)}{v}=b_{(m)} \cdot \stackrel{(\alpha)}{v}_{(m)}, \quad \stackrel{(\alpha)}{N}_{(m)}=(E A / L) \stackrel{(\alpha)}{e}_{(m)}, \quad \text { and } \stackrel{(\alpha)}{v}_{(m)}
$$

is the difference in the buckling mode displacements for mode $\alpha$ at each end of member $m$.

Expanding eqns (30) in terms of the global and local modes, and exploiting the sparsity of the postbuckling coefficients described by eqns (33)-(36) gives 


$$
\begin{gathered}
-2 \lambda_{1} \alpha_{\alpha}+\sum_{j \in M} \phi_{\alpha j j}\left(\alpha_{j}\right)^{2}+\sum_{\beta, \gamma \in G} \phi_{\alpha \beta \gamma} \alpha_{\beta} \alpha_{\gamma}=0 \quad \forall \alpha \in G, \\
\alpha_{i}\left(-\lambda_{1}+\sum_{\beta \in G} \phi_{i i \beta} \alpha_{\beta}\right)=0 \quad \forall i \in M
\end{gathered}
$$

Of particular interest is the real solution to this system for which $\lambda_{1}$ is a minimum, since this corresponds to the postbuckling branch on which the lead drops most rapidly, and also provides the worst imperfection shape (Koiter, 1976; Triantafyllidis and Peek, 1992). This most critical postbuckling branch is not necessarily unique. To find it (or them), the following constrained minimization problem needs to be solved :

$$
\begin{array}{cc}
\text { Minimize } & 2 \lambda_{1}=\sum_{I, J, K \in G \cup M} \phi_{I J K} \alpha_{l} \alpha_{J} \alpha_{K} \\
\text { subject to } & \sum_{l \in G \cup M}\left(\alpha_{I}\right)^{2}=1 .
\end{array}
$$

By setting $y_{i} \equiv\left(\alpha_{i}\right)^{2}$ for all $i \in M$, expanding eqns (39)-(40) into its contributions from local and global modes, and using eqns (33)-(36) the above minimization problem reduces to:

Minimize

$$
2 \lambda_{1}=\sum_{\alpha, \beta, \gamma \in G} \phi_{\alpha \beta \gamma} \alpha_{\alpha} \alpha_{\beta} \alpha_{\gamma}+3 \sum_{i \in M}\left(\sum_{\alpha \in G} \phi_{i i \alpha} \alpha_{\alpha}\right) y_{i}
$$

Subject to

$$
\sum_{\alpha \in G}\left(\alpha_{\alpha}\right)^{2}+\sum_{i \in M} y_{i}=1, \quad y_{i} \geqslant 0
$$

Clearly both the objective function [eqn (41)] as well as the constraint conditions [eqns (42)] are linear in $y_{i}$. As a result, minimization with respect to $y_{i}$ for a given $\alpha_{\alpha}$ is particularly simple: the only nonzero $y_{i}$ is the one for which the coefficient in parentheses in eqn (41) is a minimum. (Unless there is more than one value of $i$ for which the coefficient in parentheses in eqn (41) attains a minimum, in which case the minimizing solution is not unique, and can involve one or more nonzero $y_{i}$.) This means that a critical postbuckling branch can always be found that involves buckling of not more than one member. For the case that only one member, $i=p$, buckles, the leading order equilibrium equations [eqns (37)-(38)] reduce to

$$
\phi_{p p \alpha}+\sum_{\beta, \gamma \in G} \bar{\phi}_{\alpha \beta \gamma} \alpha_{\beta} \alpha_{\gamma}=0
$$

where

$$
\bar{\phi}_{\alpha \beta \gamma}=\phi_{\alpha \beta \gamma}-\phi_{p p \alpha} \delta_{\beta \gamma}-\phi_{p p \beta} \delta_{\alpha \gamma}-\phi_{p p \gamma} \delta_{\alpha \beta} .
$$

Once the solution to the nonhomogeneous equation (43) has been obtained, the member buckling displacement $y_{p}$ and the parameter $\lambda_{1}$ are recovered from

$$
y_{p}=1-\sum_{\alpha \in G}\left(\alpha_{\alpha}\right)^{2}, \quad \lambda_{1}=\sum_{\alpha \in G} \phi_{p p \alpha} \alpha_{\alpha} .
$$

Only real solutions are retained; solutions with negative $y_{p}$ involve complex $\alpha_{p}$ and are therefore rejected.

All solutions to eqns (43) can be found numerically using homotopy methods (Watson et al., 1987). There are $2^{n(G)-1}$ pairs of solutions, $\pm \alpha_{\alpha}$, including the complex ones. Essentially, the homotopy approach involves starting with a similar problem for which the solutions are known and then gradually changing the problem into the one for which the solutions are sought while tracking the solutions numerically in the process. Unfortunately it cannot be known in advance whether a particular solution path will lead to a real solution 
or not; all solutions must be tracked even though only the real ones are retained. This means that the computational effort of finding the most critical postbuckling branch and worst imperfection shape is exponentially increasing with the number of global modes $n(G)$ present, but only linearly increasing with the number of local modes $n(M)$.

\section{STABILITY OF BIFURCATED EQUILIBRIUM BRANCHES FOR PERFECT STRUCTURE}

The stability of the bifurcated equilibrium branches in the vicinity of the bifurcation points depends on the matrix of coefficients

$$
B_{I J}=-\lambda_{1} \delta_{I J}+\sum_{K \in G \cup M} \phi_{I J K} \alpha_{K}
$$

If, for any values of the real coefficients, $Y_{I}$, the stability quantity

$$
B=\sum_{I, J \in G \cup M} B_{I J} Y_{I} Y_{J}
$$

can be made negative, the equilibrium branch [described by eqns (29)] is unstable for $\xi>0$; if $B$ can be made positive the equilibrium branch is unstable for $\xi<0$. Both these conditions are met when $B_{I J}$ is indefinite, in which case both sides of the branch are unstable. The descending side of the branch is always unstable. If $B_{I J}$ is singular and semidefinite, higher order terms need to be considered.

In evaluating this incremental stability matrix it is hepful to distinguish not only between local and global modes, but also between local modes for members that buckle $i \in M_{1}=\left\{m \in M: \alpha_{m} \neq 0\right\}$, and other local modes $i \in M_{0} \equiv\left\{m \in M: \alpha_{m}=0\right\}$, corresponding to members that do not buckle for the bifurcated branch under consideration. The submatrices in the resulting partition of the incremental stability matrix are:

$$
\begin{array}{ll}
B_{i J}=B_{J i}=\left(-\lambda_{1}+\sum_{\beta \in G} \phi_{i i \beta} \alpha_{\beta}\right) \delta_{i J} & \forall(i, J) \in M_{0} \times(M \cup G), \\
B_{i j}=0 & \forall(i, j) \in M_{1}^{2}, \\
B_{i \beta}=B_{\beta i}=\phi_{i i \beta} \alpha_{i} & \forall(i, \beta) \in M_{1} \times G, \\
B_{\alpha \beta}=-\lambda_{1} \delta_{\alpha \beta}+\sum_{\gamma \in G} \phi_{\alpha \beta \gamma} \alpha_{\gamma} & \forall(\alpha, \beta) \in G^{2} .
\end{array}
$$

A number of interesting conclusions can be drawn from eqns (48):

(i) If the term in parentheses in eqn (48a) is negative for some $i \in M_{0}$, the incremental stability matrix has an eigenvector $Y_{J}=\delta_{i J}$ for which the corresponding eigenvalue is negative. This means that member $i$ is locally unstable, a condition that is only possible if the axial compression in the member exceeds the member buckling load, while the postbuckling branch does not involve buckling of the member. Such postbuckling branches will be referred to as locally unstable. The condition for local stability is

$$
-\lambda_{1}+\sum_{\beta \in G} \phi_{i i \beta} \alpha_{\beta} \geqslant 0 \quad \forall i \in M_{0} .
$$

The Kuhn-Tucker conditions (Gill et al., 1981) for the minimization problem described by eqns (41) and (42), lead to the conclusion that eqn (49) is satisfied for the critical postbuckling branch(es). Thus the postbuckling branch(es) on which the load drops most rapidly is (are) locally stable.

(ii) Evaluating the stability quantity $B$ of eqn (47) with $Y_{i}=0 \forall i \in M_{0}$ and $Y_{\alpha}=\alpha_{\alpha}$ $\forall \alpha \in G$, and using eqn (38) produces 


$$
B=2 \lambda_{1} \sum_{i \in M_{1}} \alpha_{i} Y_{i}+\sum_{\alpha, \beta \in G} B_{\alpha \beta} \alpha_{\alpha} \alpha_{\beta}
$$

from which it can be seen that as long as $\lambda_{1} \neq 0$, and $M_{1}$ is nonempty the sign of $B$ can be controlled by suitable choice of $Y_{i}, i \in M_{1}$. Thus all bifurcated branches involving local buckling with $\lambda_{1} \neq 0$ are unstable. This includes the ascending as well as the descending side of each branch. On the other hand if $\lambda_{1}=0$, the stability matrix is singular, indicating that higher order terms need to be investigated before the stability of the branch can be assessed.

(iii) If the number of active local modes, $n\left(M_{1}\right)$, and the number of global modes, $n(G)$, coincide, and the square matrix $\phi_{i i \beta},(i, \beta) \in M_{1} \times G$ is nonsingular, then the solution is nonsingular. Finding such a solution requires solving only linear equations: take $\lambda_{1}=1$; solve the system

$$
-\lambda_{1}+\sum_{\beta \in G} \phi_{i i \beta} \alpha_{\beta}=0 \quad \forall i \in M_{1}
$$

[deduced from eqn (38)] for $\alpha_{\beta}$; then solve eqn (37) for $y_{j}=\left(\alpha_{j}\right)^{2}$, and rescale the solution to satisfy eqn (31). If not all $y_{j}$ calculated by this procedure have the same sign, the solution is complex and should be discarded.

(iv) Solutions in which the number of members that buckle, $n\left(M_{1}\right)$, exceeds the number of global modes available, $n(G)$, are only possible in pathological cases, since in this case eqn (51) involves more equations than unknowns. Furthermore, any such solutions are singular, and nonisolated. $\dagger$.

\section{IMPERFECTIONS AND THEIR PROJECTIONS}

The imperfections considered are of three types: changes in joint coordinates, $\bar{v}$, imperfections in member length $\bar{e}$, and imperfections in straightness of the members $\bar{W}$. A complete description of the imperfections of the structure is denoted by $\bar{u}=(\bar{v}, \bar{e}, \bar{W})$.

More specifically, the imperfect structure is assumed to have a reference configuration in which the coordinates of the joints are $\bar{X}=X+\bar{v}$. The length of a member in this reference configuration for the imperfect structure is denoted by $\bar{L}$. However, the reference configuration need not be stress free. The stress free length of the member (denoted by $L$ ) differs from $\bar{L}$ by an initial elongation, $\bar{e}_{(m)} \equiv L-\bar{L}$, where $m$ denotes the member number. (A subscript $(m)$ is also added to the member lengths $L, \bar{L}$ and $l$ where it is not obvious from the context which member is being referred to.) The collection of all initial member elongations, $\bar{e}_{(m)}$ is denoted by $\bar{e}$.

The initial out-of-straightness for member $m$ is described by a function $\bar{W}_{(m)}(\cdot)$, defined such that the geometry of the member centroidal line in its stress-free condition is given by $\tilde{y}=\bar{W}_{(m)}(\tilde{x} / L)$, where $(\tilde{x}, \tilde{y})$ are local coordinates for which the origin is at one of the end points of the member, and the $\tilde{x}$-axis points towards the other end point. End eccentricities can be represented by $W_{(m)}$ functions which have nonzero values at the end points. The collection of all functions $\bar{W}_{(m)}$ is denoted by $\bar{W}$ [i.e. $\left.\bar{W}=\left(\ldots, \bar{W}_{(m)}, \ldots\right)\right]$.

Suppose the imperfection $\bar{u}$ is written as $\bar{u}=\varepsilon \bar{u}$, where $\bar{u}=(\bar{v}, \bar{e}, \bar{W})$ is the imperfection shape, and $\varepsilon$ denotes the magnitude of the imperfection. Suppose further that the imperfection shape is normalized so that

$$
\sum_{I \in G \cup M}\left(\xi_{l}\right)^{2}=1
$$

where $\bar{\xi}_{I}$ are projections of the imperfection given by

\footnotetext{
$†$ More precisely, the solutions to the leading order equilibrium equations are nonisolated. Including higher order terms could still lead to isolated solutions.
} 


$$
\bar{\xi}_{I}=-\frac{1}{\lambda_{\mathrm{c}} \phi_{0}} \phi_{, u \bar{u}}^{\mathrm{c}} \bar{u}_{u}^{(l)}
$$

For small magnitudes of the imperfection $\varepsilon$, the load drop for a given magnitude of imperfection, depends only on the projections $\xi_{l}$. According to general results regarding the worst imperfection shape, the largest load drop, for a given magnitude of imperfection occurs for $\xi_{I}=\alpha_{I}$, where $\alpha_{I}$ are the solutions to eqns (30) and (31) [or, equivalently, eqns (31), (37) and (38)] for which $\lambda_{1}$ is the smallest (i.e. most negative). For such an imperfection of the worst shape, the relationship between the drop in load-carrying capacity $\Delta \lambda$ and the imperfection amplitude $\varepsilon$ is

$$
\left(\frac{\Delta \lambda}{\lambda_{\mathrm{c}}}\right)=2\left(\frac{-\lambda_{1} \varepsilon}{\lambda_{\mathrm{c}}}\right)^{1 / 2}+O(\varepsilon)
$$

Evaluating the projections of eqn (53) for the local and global modes gives

$$
\begin{aligned}
& \xi_{\alpha}=-\frac{1}{\lambda_{\mathrm{c}} \phi_{0}}\left\{\phi_{, v \bar{v}}^{\mathrm{c}} \bar{v}+\phi_{, v e}^{\mathrm{c}} \bar{e}+\phi_{, v W}^{\mathrm{c}} \bar{W}\right\}^{(\alpha)} \quad \forall \alpha \in G, \\
& \left.\xi_{i}=-\frac{1}{\lambda_{c} \phi_{0}}\left\{\phi_{, w \bar{v}}^{\mathrm{c}} \overline{\bar{v}}+\phi_{, w \bar{e}}^{\mathrm{c}} \bar{e}+\phi_{, w \bar{W}}^{\mathrm{c}} \bar{W}\right\}\right\}_{W}^{(i)} \quad \forall i \in M .
\end{aligned}
$$

It follows from symmetries of the potential energy function $\phi((v, w), \lambda,(\bar{v}, \bar{e}, \bar{W}))$ with respect to $w$ (when $\bar{W}=0$ ), and with respect to $\bar{W}($ when $w=0)$ that $\phi_{. w \bar{c}}^{\mathrm{c}}=\phi_{. w \bar{e}}^{\mathrm{c}}=\phi_{., v W}=0$ on the principal equilibrium branch. This result, together with eqns (26) and (27), and results for the derivative of the member potential energy with respect to $\bar{W}_{(m)}$ given in Peek and Triantafyllidis (1992), Appendix B allow eqns (55) and (56) to be reduced to

$$
\begin{aligned}
& \xi_{\alpha}=-\frac{1}{\lambda_{c} \phi_{0}}\left\{\phi_{, v \bar{v}} \bar{v}+\phi_{, v e} \bar{e}\right\}^{(\alpha)} \quad \forall \alpha \in G, \\
& \xi_{i}=\frac{N_{\mathrm{c}(i)} \bar{w}_{(i)}}{\sqrt{-\lambda_{\mathrm{c}} \dot{N}_{(i)} N_{\mathrm{c}(0)} L_{(i)} L_{(0)}}} \quad \forall i \in M,
\end{aligned}
$$

where

$$
\bar{w}_{(i)}=2 \int_{0}^{1} \sin (\pi \eta) \bar{W}_{(i)}(\eta) \mathrm{d} \eta
$$

is the amplitude of an equivalent sinusoidal imperfection for member $i$, and the energy derivatives in eqn (57) are given by

$$
\begin{aligned}
& \phi_{v v \bar{v}}^{\mathrm{c}} \bar{v}^{(\alpha)}=\sum_{m}\left\{\frac{E A}{L}\left(\frac{v_{(m)}}{l}-\frac{\bar{e}_{(m)}}{l} \bar{B}_{(m)}\right)-N\left[\bar{B}_{(m)}\left(\frac{1}{L}+\frac{1}{l}\right)+\frac{b_{(m)}}{l}\right]\right\}_{(m)} \\
& \cdot \bar{v}_{(m)} \stackrel{(\alpha)}{e}_{(m)}+\left(\frac{N}{l}\right)_{(m)} \bar{v}_{(m)} \cdot{\stackrel{(\alpha)}{v_{(m)}}}^{(1)} \\
& \phi_{, v e \bar{e}} e^{-(\alpha)} v=-\sum_{m}\left(\frac{l E A}{L^{2}}\right)_{(m)} \stackrel{(\alpha)}{e}_{(m)},
\end{aligned}
$$

where $\bar{B}_{(m)} \equiv \bar{X}_{(m)} / \bar{L}$ is a unit vector along the member direction for member $m$ in the reference configuration for the imperfect structure. 


\section{EXAMPLES}

\subsection{Description of structures considered}

To illustrate the asymptotic analysis described above, the critical bifurcated branches are determined for the planar truss of Fig. 1, and for the three-dimensional lattice columns of triangular and square cross-section shown in Fig. 2. These structures are identical to the ones considered in Peek and Triantafyllidis (1992), except that certain dimensions differ: all members have the same length $L$, except for the diagonal members, which are of length $\sqrt{2} L$. The axial rigidity $E A$ is the same for all members. (The actual values for $E A$ and $L$ do not affect the non-dimensional results presented here.) Dashed lines represent members in tension, whereas continuous lines are members in compression. The flexural rigidity $E I$ for the members in compression is chosen so that all such members reach their buckling load at $\lambda=\lambda_{\mathrm{c}}$, where $\lambda_{\mathrm{c}}$ is also the load for which the global buckling modes develop. Although for the sake of clarity, Figs 1 and 2 show only two bays, all the trusses analysed here have 10 bays, unless otherwise noted. The numbering of the joints follows the pattern established in Figs 1 and 2.

The boundary conditions for the planar truss are as shown in Fig. 1. For the threedimensional trusses of Fig. 2, the axial $z$-component of displacement is zero for joints $A_{0}$, $B_{0}, C_{0}$ and $D_{0}$. In addition, the minimal constraints required to prevent rigid body motions are provided in the $x$ - and $y$-directions.

In order to simplify the prebuckling solution, the structures are constructed from identical unit cells. Thus member sites perpendicular to the axis of loading (except those at the ends of the structure) are occupied by two identical members. This results in a prebuckling stress state that does not change from one unit cell to the next.

\subsection{Results}

For the planar truss of Fig. 1, a single global buckling mode (mode number 1) develops at $\lambda_{\mathrm{c}} / E A=0.00614$. The global-global postbuckling coefficient $\phi_{111}$ is zero, since in the absence of local modes, the bifurcation is symmetric. Only solutions involving buckling of a single member are sought, since the critical bifurcated branch must have this property. For this purpose, eqns (43)-(45) lead to

$$
\alpha_{1}= \pm \frac{1}{\sqrt{3}}, \quad \alpha_{p}= \pm \sqrt{\frac{2}{3}}, \quad \lambda_{1}=\phi_{p p 1} \alpha_{1},
$$

where $p$ is the identifier for the member that is assumed to buckle. It follows from eqn (62) that the critical bifurcated branch (that for which $\lambda_{1}$ is smallest) involves buckling of the
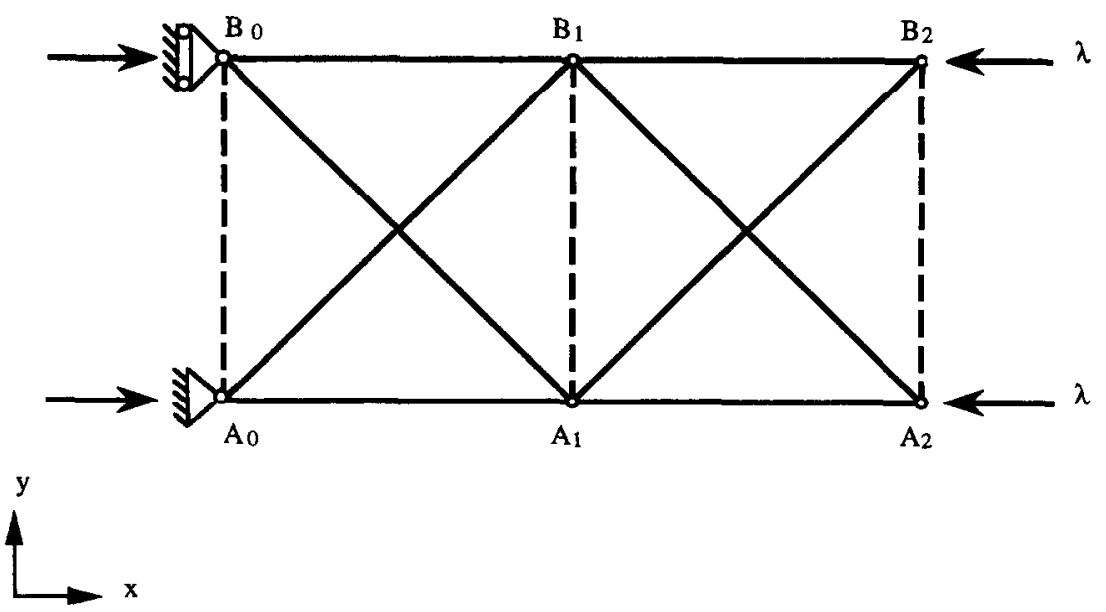

Fig. 1. Planar truss. 


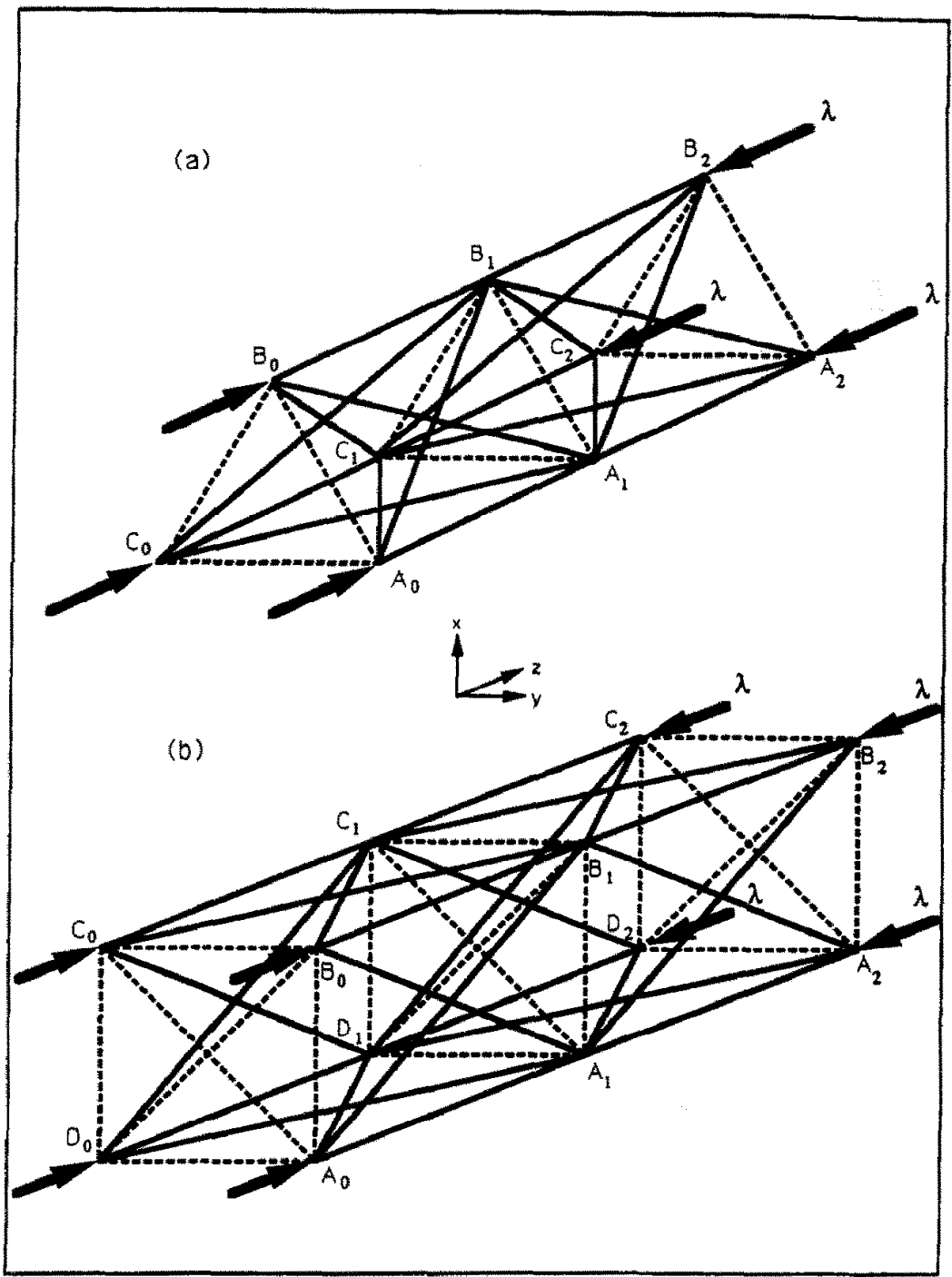

Fig. 2. (a) Triangular, and (b) square lattice columns.

member $p$ for which the local-global interaction coefficient $\phi_{p p}$ is largest in absolute value. This leads (not surprisingly) to the result that the critical branch involves buckling of chord member $A_{0}-A_{1}$ or $B_{0}-B_{1}$ only.

The results for the planar truss are shown in Figs 3 and 4, for the bifurcated branch involving buckling of member $B_{0}-B_{1}$. Therein the asymptotic results are compared to exact results obtained by numerically tracking the bifurcated branches, as is described in the Appendix. The joint displacement plotted is the $y$-component of the displacement at joint $A_{10}$. Figure 4 shows the member buckling displacements for all members that buckle at any particular value of the joint displacement. For the exact solution this member buckling displacement is obtained from the elastica solution as the transverse displacement at midspan from a straight line connecting the end points of the member. This coincides with the member buckling displacement $w_{(m)}$ introduced in eqn (2), except for higher order terms.

In all cases the asymptotic and exact solutions coincide sufficiently close to the bifurcation point, as they should. (This was ascertained from magnified views of the plots of the bifurcated branches in the vicinity of the bifurcation point, which are not shown here.) Away from the bifurcation point, some differences become apparent, since the asymptotic analysis includes only the leading order terms. 


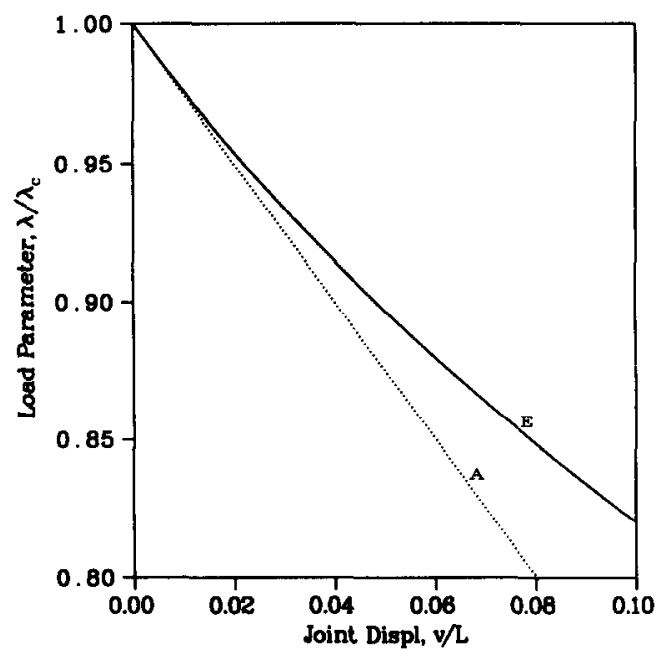

Fig. 3. Load versus transverse joint displacement for planar truss: (A) asymptotic result, (E) exact result.

Whereas initially, only the first chord member buckles, as predicted by the asymptotic result, Fig. 4 indicates that the next chord member buckles soon thereafter (at a joint displacement of $v / L \equiv 0.01$ ), which is then followed by buckling of the third chord member at $v / L \cong 0.05$. Pursuing the bifurcated branch for joint displacements larger than those shown in Fig. 4, leads to buckling of the fourth chord member at $v / L \cong 0.17$, and buckling of a diagonal member $B_{0}-A_{1}$ at $v / L \cong 0.23$.

Before the second chord member buckles, the agreement between the exact and asymptotic results is very good. The buckling of the second chord member in itself is a deviation from the asymptotic results (which predict zero buckling displacement for the second chord member). In addition, there is a change in slope in member buckling displacement curve for the first member, when the second member buckles. Thereafter, the differences between the exact and asymptotic results for the first member increase. When the third chord member buckles, there is again a change in slope in the curves for the first and second members leading to more rapidly increasing differences between the exact and asymptotic results for the first chord member.

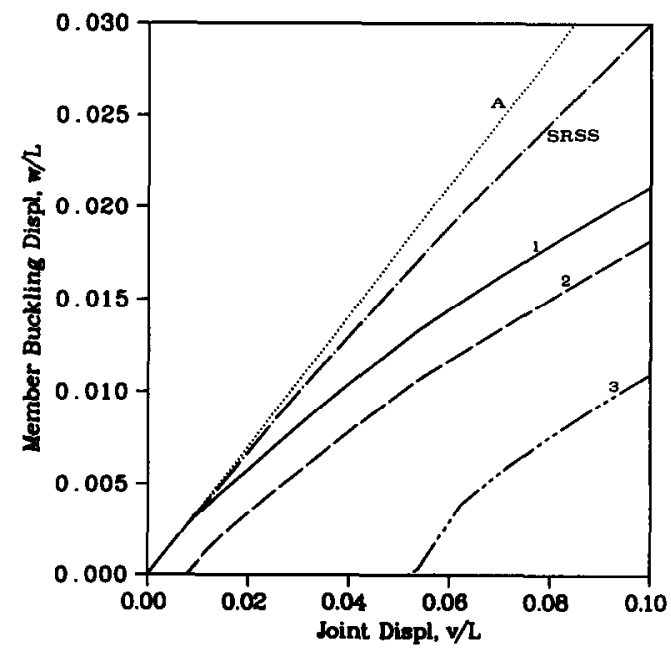

Fig. 4. Member buckling displacements for planar truss : (A) asymptotic result for member $B_{0}-B_{1}$, (1,2 and 3) exact results for members $B_{0}-B_{1}, B_{1}-B_{2}, B_{3}-B_{4}$ and $B_{4}-B_{5}$ respectively, (SRSS) square root of the sum of the squares buckling displacements for all members on chord $B$. 


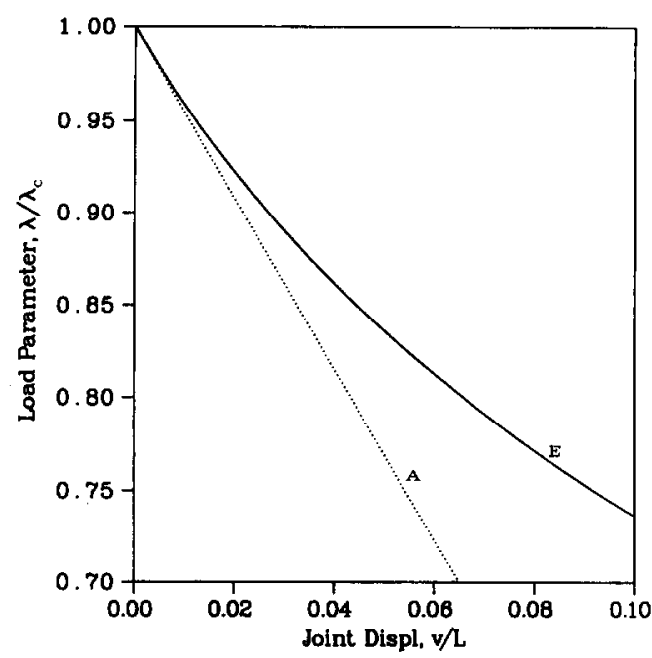

Fig. 5. Load versus transverse joint displacement for triangular lattice column: (A) asymptotic result, (E) exact result.

The member shortening is approximately proportional to the member buckling displacement squared. Thus, if the total shortening of the chord due to buckling of all chord members were to be accommodated by buckling of the first chord member only, the member buckling displacement for the first chord member would be approximately the square root of the sum of the squares (SRSS) of the member buckling displacements for all chord members that buckle. As seen from Fig. 4, this SRSS member buckling displacement falls close to the asymptotic result. Thus the asymptotic result provides a good approximation for the total shortening of chord due to buckling of the members, even when there are substantial differences in the buckling displacements of individual members.

Similar results appear in Figs 5-8 for the three-dimensional trusses. In this case symmetries lead to two coincident global buckling modes at $\lambda_{\mathrm{c}} / E A=0.00463$ for the lattice column of triangular cross-section, and $\lambda_{\mathrm{c}} / E A=0.00768$ for that of square cross-section. Also certain global-global postbuckling coefficients $\left(\phi_{\alpha \beta \gamma}, \alpha, \beta, \gamma \in G\right)$ are nonzero. Thus eqns (43) lead to two coupled quadratic equations in the global buckling displacements. All the solutions to this system (including the complex ones, which are rejected since they have no physical meaning) were calculated using the homotopy algorithms developed by

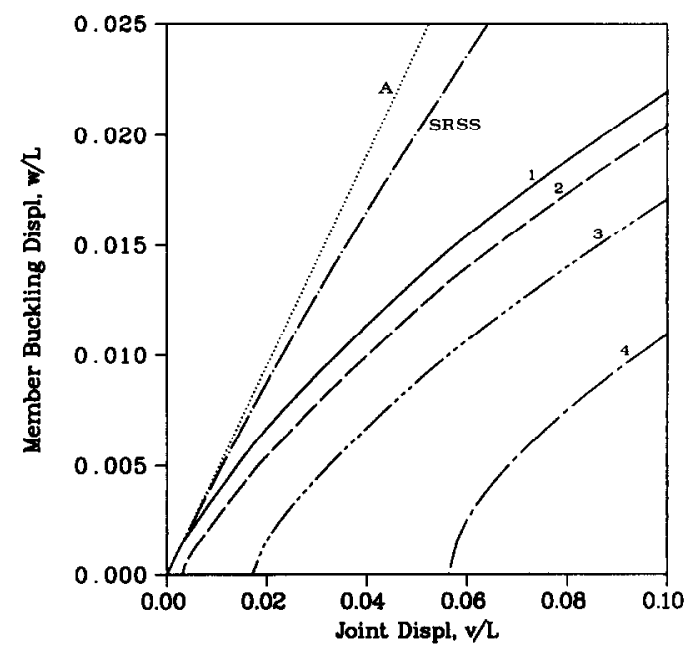

Fig. 6. Member buckling displacements for triangular lattice column: (A) asymptotic result for member $B_{0}-B_{1},\left(1,2,3\right.$ and 4) exact results for members $B_{0}-B_{1}, B_{1}-B_{2}, B_{3}-B_{4}$ and $B_{4}-B_{5}$ respectively, (SRSS) square root of the sum of the squares exact buckling displacements for all members on chord $B$. 


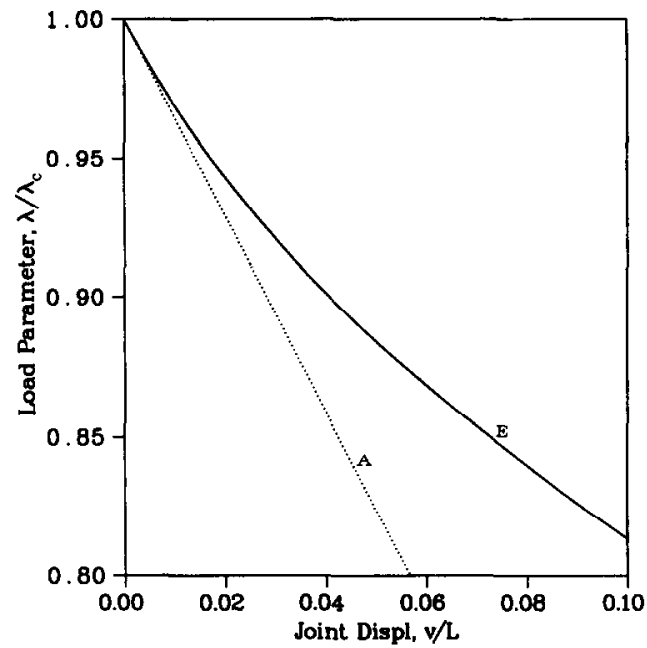

Fig. 7. Load versus transverse joint displacement for square lattice column: (A) asymptotic result, (E) exact result.

Watson et al. (1987). This process was repeated for every potentially buckling member, $p$, leading to the conclusion that the critical bifurcated branch involves buckling of one of the first chord members (e.g. member $B_{0}-B_{1}$ ).

Whereas the manner in which joint displacements in the $x-y$ plane are restrained does not influence the postbuckling behavior, it does have a small influence on the joint displacements, since some deformations of the cross-section do occur.

For the triangular cross-section, joints $A_{0}$ and $C_{0}$ are restrained in the $x$-direction, and joint $B_{0}$ is restrained in the $y$-direction. The critical branch considered is the one for which member $B_{0}-B_{1}$ buckles. Thus symmetry of the postbuckling solution about the $x-z$ plane is preserved. The joint displacement of Figs 5 and 6 is the $x$-component of displacement at joint $A_{10}$ (or $C_{10}$ ).

For the square cross-section, joint $D_{0}$ is restrained in both the $x$ - and $y$-directions, and joint $A_{0}$ is restrained in the $x$-direction. The critical branch involving buckling of member $B_{0}-B_{1}$ is considered, and the joint displacement plotted in Figs 7 and 8 is the magnitude of the projection of the displacement at joint $D_{10}$ onto the $x-y$ plane.

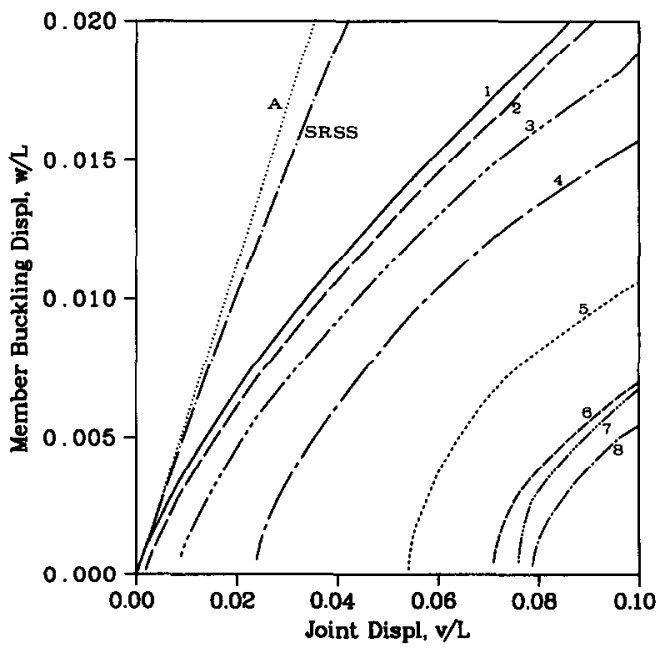

Fig. 8. Member buckling displacements for triangular lattice column : (A) asymptotic result for member $B_{0}-B_{1},(1-5)$ exact results for chord members $B_{0}-B_{1}$ through $B_{4}-B_{5}$, respectively, (68) exact results for diagonal members $B_{0}-A_{1}$ and $B_{0}-C_{1}, B_{1}-A_{2}$ and $B_{1}-C_{2}$ and $C_{0}-B_{1}$ and $A_{0}-B_{1}$, respectively, (SRSS) square root of the sum of the squares exact buckling displacements for all members on chord $B$. 
The behavior for these three-dimensional lattice columns is very similar to that for the planar truss. The four-chord lattice column is somewhat less imperfection sensitive than the three-chord column. This might be expected in view of greater redundancy for the fourchord truss. It can also be observed that buckling of the diagonal members begins at lower joint displacements for the four-chord truss. Furthermore, those diagonal members for which the cross-sectional shear force produces compression buckle earlier.

In order to investigate the effect of slenderness of the structures, a 25-bay square crosssection lattice column made from unit cells identical to those for the corresponding 10-bay column was analysed, except that the flexural rigidity of the members $E I$ is adjusted so as to maintain coincidence of the local and global modes. The postbuckling behavior was found to be essentially identical to the 10-bay column: buckling of the first chord member is followed by buckling of additional chord members until members half way up the chord have buckled. (Of course, this involves the buckling of a larger number of members for the 25 -bay column.) Then buckling of diagonal members begins. The plot of the load parameter $\lambda / \lambda_{\mathrm{c}}$ as a function of joint displacement $v / L$ for the 25-bay column (not shown here) is essentially indistinguishable from that for the 10-bay column.

The buckling of additional members also merits some discussion. This represents a secondary bifurcation of the system as a whole. If a single member buckles, a simple secondary bifurcation is at hand. The continuation of the branch leading to the secondary bifurcation involves local instability for the member that buckles, and is therefore of no interest. Since the buckling of a single member is symmetrical, both sides of the secondary bifurcated branch are identical. This means that the continuation of the equilibrium branch onto the secondary bifurcated branch is unique, and can be determined by the analysis procedure described in the Appendix.

For buckling of the diagonal members of the three-dimensional structures a different situation arises, however. Due to symmetry, two diagonal members always reach their buckling load simultaneously. This gives rise to a multiple secondary bifurcation involving local modes only. Thus, there could be three locally stable secondary bifurcated equilibrium branches : one involving the buckling of one of the members, one involving the buckling of the other member, and one involving the buckling of both members. For all cases considered here, the solution procedure described in the Appendix converged to the solution involving the buckling of both diagonal members. Whether the other secondary bifurcated branches exist as real, locally stable solutions is not established in the present analysis. To establish this, a local-local mode interaction analysis such as that performed in the companion paper (Peek and Triantafyllidis, 1992) would need to be performed. Thus once the diagonal members buckle, the results for the three-dimensional trusses need to be looked at with caution, since other solution branches may also be present.

Finally it is of interest to compare the present results with those obtained by Luongo and Pignataro (1988) for local global mode interactions in the buckling of thinwalled members under axial compression. They found that the only nonzero postbuckling coefficients are $\phi_{1, j}$ where 1 represents the global mode and $i, j$ represent local modes. As a result, they were able to obtain bifurcated equilibrium branches by solving an eigenvalue problem. They also deduced that one third of the change in total potential energy from the principal equilibrium branch to the bifurcated branch (at the same level of the load parameter $\lambda$ ) is due to the global mode, whereas two thirds is due to the local modes.

For the planar truss example considered here, there is only one global buckling mode, and the only nonzero postbuckling coefficients are $\phi_{1 i i}$. The structure of nonzero postbuckling coefficients is thus a special case of that considered by Pignataro and Luongo. As a result, the one third global and two thirds local split of the energy also applies here. Indeed it can readily be shown [from eqns (37) and (38)] that this energy split also applies here for the case when several global modes are considered, provided that in the absence of local modes the bifurcation is symmetric (i.e. provided that $\phi_{\alpha \beta \gamma}=0 \forall \alpha, \beta, \gamma \in G$ ). This condition is satisfied for the lattice column of square cross-section, but not for that of triangular cross-section. Consistent with this, numerical values of the fraction of the energy change attributable to global modes is $1 / 3$ (to 12 digits of accuracy) for the lattice column of square cross-section, and 0.33309 for that of rectangular cross-section. Thus, even for 
the column of triangular cross-section, the one third global to two thirds local energy split of the energy is a good approximation.

In other regards the behavior of the thin walled members considered by Luongo and Pignataro differs from that of lattice structures considered here. For example, here local modes are already localized in that the single local mode involves buckling of one member only. For the thin-walled members the local modes are not localized. Rather the localization of the deformation pattern occurs as several local modes are combined, resulting in a faster drop in the load-carrying capacity of the structure. For the thin-walled member, the local modes involve different wavelengths. Therefore the critical load is not exactly the same for all local modes, and localization of the incremental deformation pattern cannot be expected to occur immediately after bifurcation, but rather a finite distance away from the bifurcation point. In contrast, for the lattice structures considered here, the deformation begins in a localized fashion immediately after bifurcation with buckling of onc member only, and the deformations spread with buckling of additional members as one moves away from the bifurcation point.

Perhaps the most important reason for these differences in postbuckling behavior of the lattice column and the thin-walled members is that for the thin-walled members continuity of the plates forming the cross-section precludes bucking modes involving a single half wave, such as are possible in the lattice column with pinned joints. Furthermore the buckling wavelength is not fixed by the geometry for the thin-walled member, whereas for the lattice structure it must be equal to the unit cell size.

\section{CONCLUSIONS}

Leading order asymptotic results have been developed for any truss-type structure with coincident local and global modes. The worst shape of imperfections is determined from the bifurcated equilibrium branch for the perfect structure on which the load drops most rapidly. It is shown that initially this critical bifurcated equilibrium branch involves the buckling of one member only. However for a number of illustrative examples involving lattice columns, an exact solution indicates that a second member buckles a small but finite distance away from the bifurcation point. Although buckling of the second member is not predicted by the leading order asymptotic results, the agreement between the asymptotic and exact results for the overall behavior of the structure is still good, well beyond the point where the second member buckles.

Acknowledgements - The author is indebted to Nicolas Triantafyllidis, who reviewed the manuscript and provided some valuable comments, as well as to Layne Watson for his help in obtaining and using his homotopy algorithms. Financial support was provided by the National Science Foundation as Grant No. CES-8857002.

\section{REFERENCES}

Forman, S. E. and Hutchinson, J. W. (1970). Buckling of reticulated shell structures. Int. J. Solids Structures 6(7), 909-932.

Gill, P. E., Murray, W. and Wright, M. H. (1981). Practical Optimization. Academic Press.

Koiter, W. T. (1976). Current trends in the theory of buckling. In Symposium on Buckling of Structures. Sponsored by the International Union of Theoretical and Applied Mechanics (IUTAM), Harvard University, Cambridge, MA, 17-24 June, 1974 (Edited by B. Budiansky), pp. 1-16. Springer, Berlin.

Luongo, A. and Pignataro, M. (1988). Multiple interaction and localization phenomena in the postbuckling of compressed thin-walled members. AIAA Jl 26(11), 1395-1417.

Peek, R. and Triantafyllidis, N. (1992). Worst shapes of imperfections for space trusses with many simultaneously buckling members. Int. J. Solids Structures 29, 2385- 2402.

Riks, E. and Rankin, C. C. (1987). Bordered equations in continuation methods : An improved solution technique. NLR MP 87057U, National Aerospace Laboratory, The Netherlands.

Timoshenko, S. P. and Gere, J. M. (1961). Theory of Elastic Stability. McGraw-Hill, Maidenhead.

Triantafyllidis, N. and Peek, R. (1992). Stability and worst imperfection shape of structures with multiple eigenmodes. Int. J. Solids Structures 29, 2281-2299.

Watson, L. T., Billups, S. C. and Morgan, A. P. (1987). HOMPACK : A suite of codes for glohally convergent homotopy algorithms. ACM Transactions on Mathematical Software 13(3), 281-310. 


\section{APPENDIX. NUMERICAL TRACKING OF BIFURCATED EQUILIBRIUM BRANCHES}

This Appendix describes the method used to track the bifurcated equilibrium branches numerically in order to obtain what are referred to as the "exact" results. Such an analysis does require prior knowledge of which member (or members) buckles for the bifurcated branch to be tracked. In the approach used, only the joint displacements $v$ as well as the load parameter $\lambda$ are used as unknowns. The need to include the member buckling displacements $w$ is eliminated by using a member force-deflection relation that includes the effect of member buckling.

To obtain this member force-elongation relation, it is assumed that the member freezes axially (i.e. becomes incompressible) when buckling occurs. This allows the elastica solution [see for instance Timoshenko and Gere (1961)] to be used in the postbuckling range. The asymptotic postbuckling analysis for the compressible column in Peek and Triantafyllidis (1992), Appendix B confirms that this is indeed a good approximation. The resulting relationship between axial force $N$, member elongation $e$ and the maximum transverse displacement $w$ (as measured from a straight line connecting the end points of the member) can be described parametrically as

$$
\frac{-N}{N_{\mathrm{c}}}=\left[\frac{2}{\pi} K(p)\right]^{2}, \quad-\frac{e}{L}=2\left[1-\frac{E(p)}{K(p)}\right], \quad \frac{w}{L}=\frac{p}{K(p)},
$$

where $p$ is a deformation parameter, which is related to the rotation at the ends of the member $\alpha$ by $p=\sin (\alpha / 2)$, and $K(\cdot)$ and $E(\cdot)$ are the complete elliptic integrals of the first and second kind, respectively, defined by

$$
K(p)=\int_{0}^{\pi / 2} \frac{\mathrm{d} \phi}{\sqrt{1-p^{2} \sin ^{2} \phi}}, \quad E(p)=\int_{0}^{\pi / 2} \sqrt{1-p^{2} \sin ^{2} \phi} \mathrm{d} \phi .
$$

Also recall that the axial force $N$ and elongation $e$ are taken to be positive when the member is in tension, whereas $N_{\mathrm{c}}$ and $e_{\mathrm{c}}$ are the absolute values of the axial force and elongation at the bifurcation point for the member. Calculating the member force $N$ for a given value of the elongation $e$ requires first solving eqn (Alb) for the parameter $p$, and then obtaining the axial force from eqn (Ala). Since such a calculation is neither convenient nor well-conditioned for small member buckling deformations, it was avoided by using the following series representation for the member force directly in terms of the elongation, derived from eqns (Ala,b) :

$$
\frac{-N}{N_{\mathrm{c}}}=1+\frac{\eta}{2}+\frac{9 \eta^{2}}{32}+\frac{21 \eta^{3}}{128}+\frac{795 \eta^{4}}{8192}+\frac{945 \eta^{5}}{16384}+\frac{2247^{6}}{65536}+\frac{42639 \eta^{7}}{2097152}+\cdots
$$

where $\eta=\left(-e-e_{\mathrm{c}}\right) / L$. To obtain the transverse displacement $w$, the following series expansion for the parameter $p$ in terms of the elongation [derived from eqn $(\mathrm{Al} / \mathrm{b})$ ] was used:

$$
p^{2}=\eta-\frac{\eta^{2}}{8}-\frac{\eta^{3}}{32}-\frac{11 \eta^{4}}{1024}-\frac{17 \eta^{5}}{4096}-\frac{55 \eta^{6}}{32768}-\cdots
$$

After calculating $p$ from eqn (A4), the transverse displacement can be calculated from eqn (Alc). In the range $|\alpha| \leqslant 0.5$, these series representations are essentially exact (maximum error in $N / N_{\mathrm{c}}$ was found to be $1.2 \times 10^{-9}$ ).

Suppose that the asymptotic analysis yields a critical bifurcated branch involving buckling of member $p$. Then, to ensure that the numerical procedure converges to the desired bifurcated equilibrium branch, the force deflection relation for member $p$ is rounded at the corner that develops at an elongation $e_{(p)}=-e_{c(p)}$ due to buckling of the member. This is done by fitting a cubic between points $e_{(p)}=-(1-\gamma) e_{c(p)}$ and $e_{(p)}=-(1+\gamma) e_{c(p)}$ (where $\gamma=0.01$ ), so that there is no discontinuity in slope of the member force-clongation rclation. This imperfection guides the numerical solution onto the chosen bifurcated branch.

Numerical tracking of the equilibrium branch is done by an incremental Newton-Raphson procedure, following a method described in Riks and Rankin (1987), in which an approximation to the arclength in loaddisplacement space is controlled at every loadstep. In addition, a quadratic predictor scheme is used to obtain the first guess for the solution at every loadstep. Specifically two points on the equilibrium branch (in load-displacement space) corresponding to loadsteps $i$ and $i-1$ as well as the direction of the equilibrium branch at loadstep $i$ are used to obtain a quadratic approximation to the equilibrium branch. In addition to using this to compute predictor values for the load and displacement at loadstep $i+1$, it is also used in an automatic step size control scheme by which the estimated change in direction of the tangent to the equilibrium branch from one loadstep to the next is not allowed to exceed 0.04 radians.

Some distance away from the bifurcation point, a state is reached in which $e_{(p)}<-(1+\gamma) e_{\mathrm{c}(p)}$. The solution for the imperfect structure described above then coincides with that for the perfect structure. After reaching such a state, the imperfection is removed, and the direction of loading reversed, to obtain the solution for the perfect structure all the way to the bifurcation point. 